\title{
Peri-implantitis as a cause of giant-cell granuloma? Presentation of a clinical case
}

\author{
Franck Afota ${ }^{1,}$, Helene Raybaud ${ }^{2}$, Alexandre Bozec ${ }^{3}$, Sarah Abid ${ }^{4}$, Charles Savoldelli ${ }^{5}$ \\ ${ }^{1}$ Oral Surgery Resident, University Institute of the Face and Neck, Oral and Maxillofacial Surgery Service, CHU Nice University, 31 Avenue de \\ Valombrose, 06100 Nice, France \\ 2 MCU-PH, Dental Service, Hospital St Roch, CHU NICE, France \\ ${ }^{3}$ PH ENT-Orofacial and Neck Surgery, University Institute of Orofacial Medicine, CHU NICE, France \\ ${ }^{4}$ Dental Student, Hospital St Roch, CHU NICE, France \\ ${ }^{5}$ MCU-PH, Oral and Maxillofacial Surgery Service, CHU NICE University Institute of the Face and neck, CHU NICE, France \\ *franck.afota@hotmail.fr
}

(Received: 4 March 2016, accepted: 11 March 2017)

Keywords:

Giant-cell granuloma /

inflammation / peri-implantitis

\begin{abstract}
Introduction: Peri implantitis can be identified by classic clinical and radiographic signs. The aim of this case was to show an original exophytic lesion appeared 8 years after the implantation in the mandibular symphysis. Observations: The patient has been implanted on symphyseal site 8 years ago. The lesion was exophytic and located around mobile implant in right mandible. First curative surgical resection was performed under local anesthesia, combined with explantation of mobile implants. Histhopathological exam identified a giants cells granuloma. A second surgical removal was necessary under general anesthesia because of a severe recurrence. Discussion: The goal of this clinical case was to emphasize the possible correlation between inflammatory peri implantits context and giants cells granuloma. Others techniques could be used to manage this case.
\end{abstract}

\section{Introduction}

Peri-implantitis is a condition encountered by implantologists on a daily basis. The classic clinical signs are implant mobility, axial percussion pain, and mucosal inflammation. There is an increase in the depth of the peri-implant, which may be accompanied by bleeding. At a radiological level, peri-implantitis results in a vertical bone defect around the neck of the implant, which can destroy the crestal bone [1]. In addition, peri-implantitis can result in the presence of a radiolucent lesion at the apex of the implant, which is suggestive of a granuloma or inflammatory cyst. The known risk factors for peri-implantitis are poor oral hygiene, malocclusion, history of periodontitis, and smoking [2]. Giant cell granuloma, also known as a reparative granuloma, is a benign lesion of the maxillae and is more commonly detected in women (3/1) than in men and at the anterior mandibular level. Osteolysis can be uni- or multilocular and has a soap bubble-like shape. Compared with expansive tumors (ameloblastomas or keratocysts) or malignant tumors, giant cell granulomas do not severely affect the maxillae. Some cases have been described in the literature [3]. This study aimed to discuss a case of a patient who developed a giant cell granuloma and peri-implantitis around mandibular implants.

\section{Observation}

Eight years ago, an 83-year-old patient consulted us for gingival swelling around her mandibular implants. The patient had no medical history, had never smoked, and had proper oral hygiene. The growth developed progressively over a year, there was no pain or neurosensory disorders. The clinical examination revealed a exophytic swelling in the oral vestibule of $2 \mathrm{~cm}$ on the implants between teeth 43 and 45 . Sector 4 Implants (42, $43,44)$ had type-2 mobility and were surrounded by an inflammatory attached gingiva (Fig. 1).

Palpation revealed a soft lesion that did not bleed on touch. There was no cervical lymphadenopathy at the level of the cervical ganglion areas or any difficulty in tongue protrusion. A computed tomography scan and dental panoramic X-ray showed radiotransparent lesions at implant apices with an osteolysis and a depression marked around the three implants in sector 4 . The implants between teeth 43 and $44 \mathrm{had}$ also migrated toward each other. The implant prosthetic system consisted of a screw in the pillar and a sealed metal-porcelain bridge (Fig. 2). There was no osteolytic lesion at the basal bone level.

The differential diagnosis included a benign tumor, a giant-cell granuloma, or a squamous carcinoma. At first, the exophytic lesion was resected under local anesthesia. The mucoperiosteal flap allowed the exposed tips of sector 4 implants to be manipulated (Fig. 3). Initial type-2 implant mobility was increased to type- 3 mobility after the narrow bridge was removed. In addition, the patient consented to for implant exploration.

Pathological analysis revealed a histological diagnosis of giantcell granulomas. There was indeed a proliferation of giant multinuclear cells in a fibrous stroma with the presence of a 


\section{SHORT CASE REPORT}

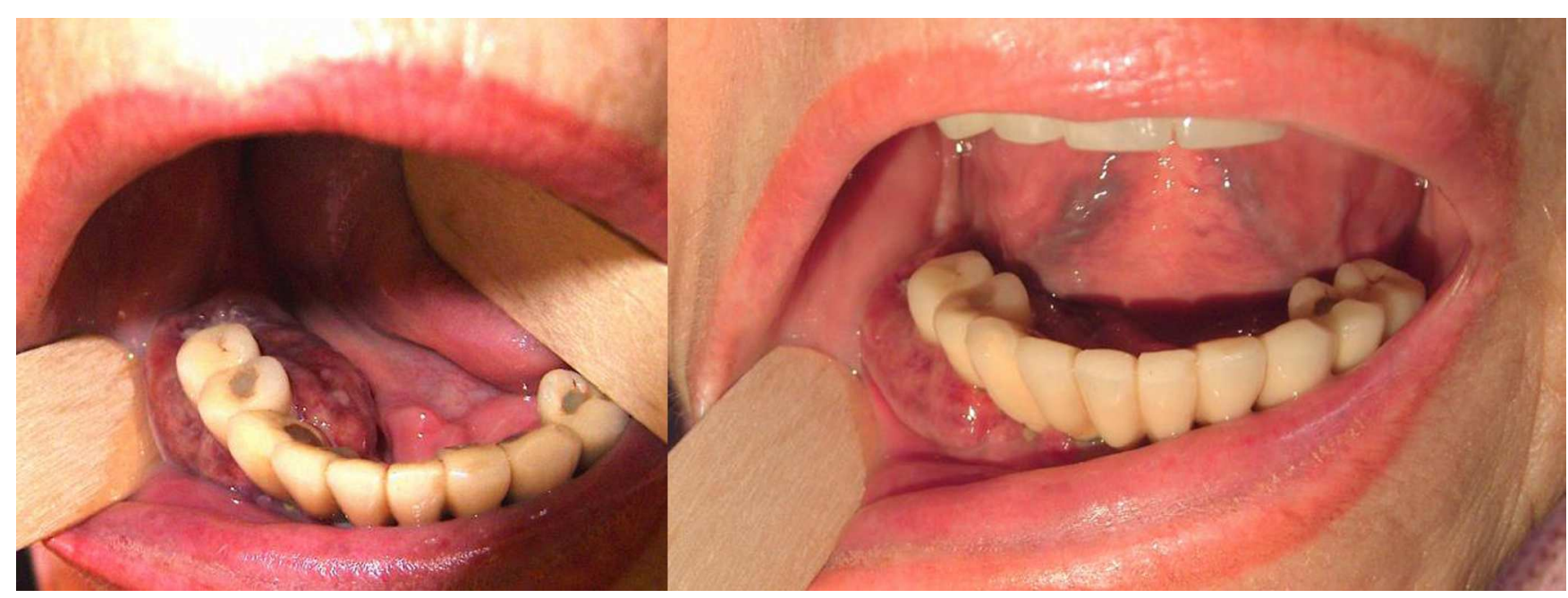

Fig. 1. Initial mandibular lesion surrounding implants in teeth 33-35.

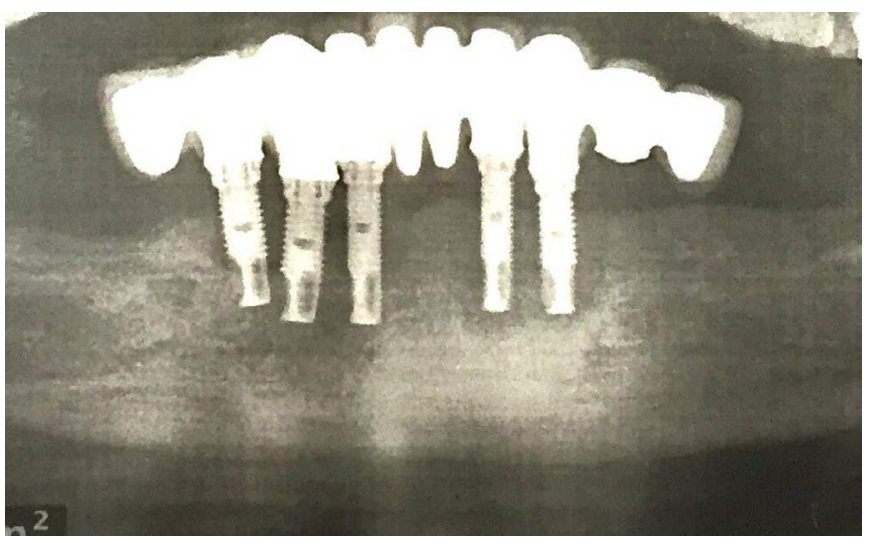

Fig. 2. Panoramic $X$-ray showing a depression around the implants placed in teeth 42,43 , and 44 .

fibrinous leukocytic exudate (Fig. 4). The postoperative treatment included antibiotic therapy (amoxicillin + clavulanic acid $3 \mathrm{~g}$ per day for 7 days), corticosteroids (prednisolone $60 \mathrm{mg}$ for 4 days), analgesics (paracetamol $3 \mathrm{~g}$ per day for 7 days), and mouthwash (sodium bicarbonate).

The postoperative clinical examination 40 days after the resection showed a recurrence of the mucosal lesion (Fig. 5). A similar growth was observed at the alveolar crest level where there were no implants.

A second procedure was performed under general anesthesia. The conditions of general anesthesia allowed a complete resection of the pedicle of the lesion and better hemostasis using thermocoagulation. We also achieved an extensive surgical curetage and a wider buccal and lingual tissue dissection for proper closure. The pathological analysis confirmed the diagnosis of giant-cell granuloma.

No clinical recurrence was detected at follow-up examinations 15 days and 2 months after the second resection, and the mucosal healing was optimal. Thereafter, the two Sector-3 implants were removed because of peri-implantitis, but there were no associated mucosal lesions. Then, we performed

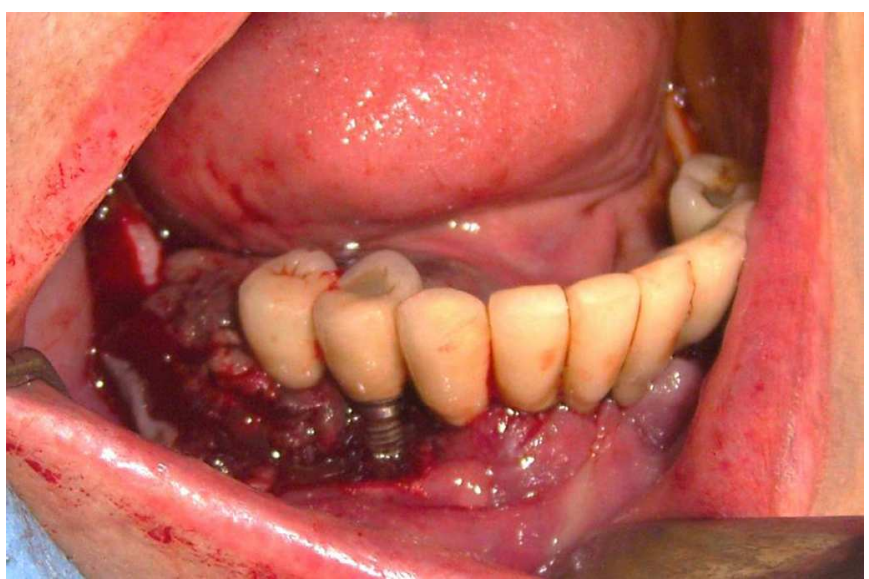

Fig. 3. Resection surgery of the lesion under local anesthesia. “The exposure of the bare tips of the implant can be observed"

prosthetic reconstruction using a removable partial denture with the installation of mini-symphyseal implants as recommended by the MacGill Consensus [3]. The lack of prosthetic stability led us to install an additional implant in addition to the two implants as recommended in the literature (Fig. 6).

\section{Discussion}

The etiopathogenesis of the giant-cell granuloma is poorly known, but the risk factors are predominantly poor oral hygiene and xerostomia [4].

The case presented showed a nonaggressive example of a mucosal giant-cell granuloma. The potential for aggressiveness of the giant-cell granuloma depends on the histological nature of the giant cells in the lesion [5]. The larger the size and the area occupied by the giant cells, the more aggressive the lesion.

In the case described, lesion recurrence was observed after the first resection. The potential for recurrence of giant-cell granuloma is well documented. The study by Lester et al. conducted on 279 giant-cell granulomas showed that 


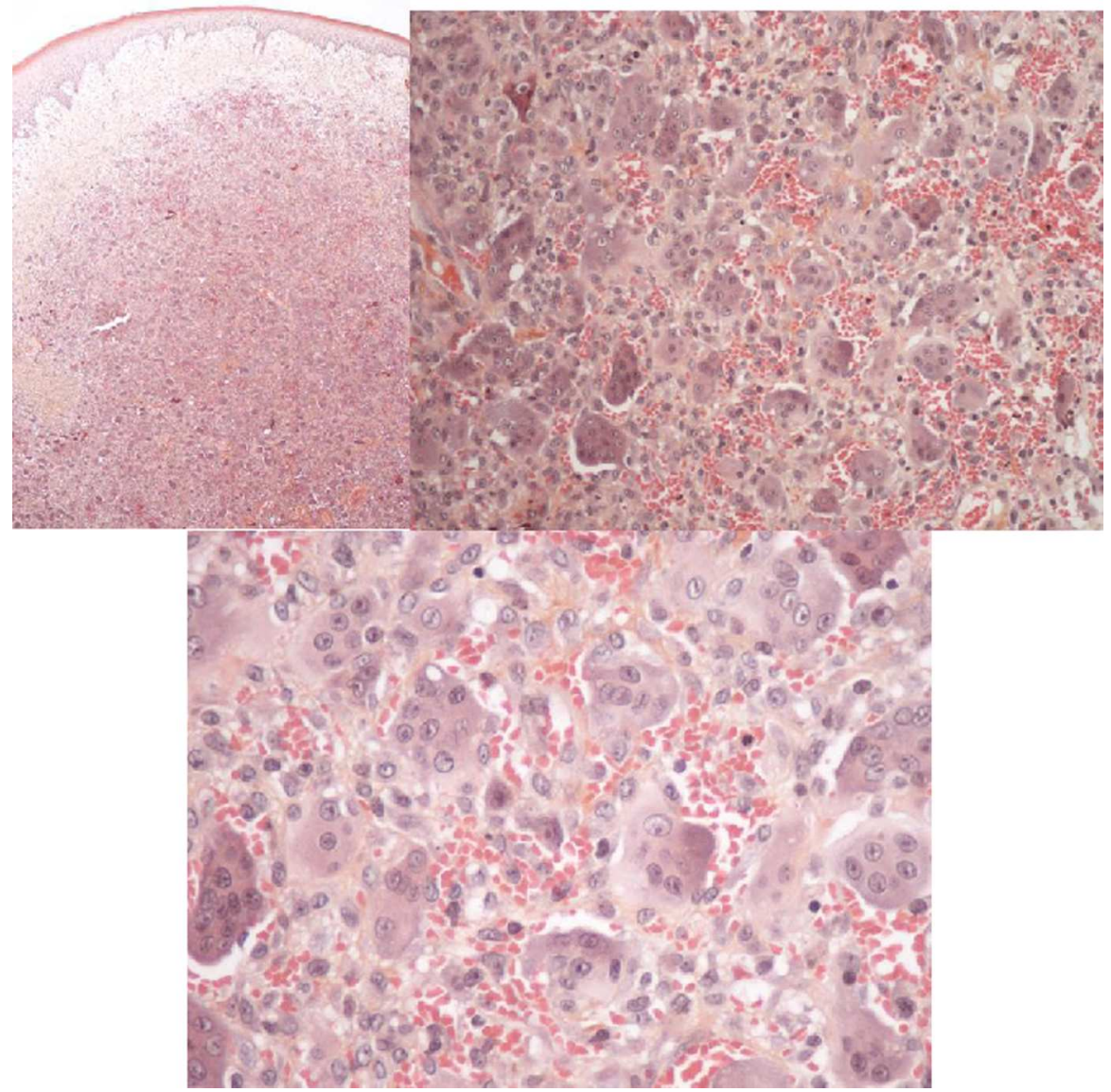

Fig. 4. Histological sections (magnification $\times 4 ; \times 20$;) showing a proliferation of giant multinuclear cells in a fibrous stroma with the presence of a fibrinous leukocytic exudate.

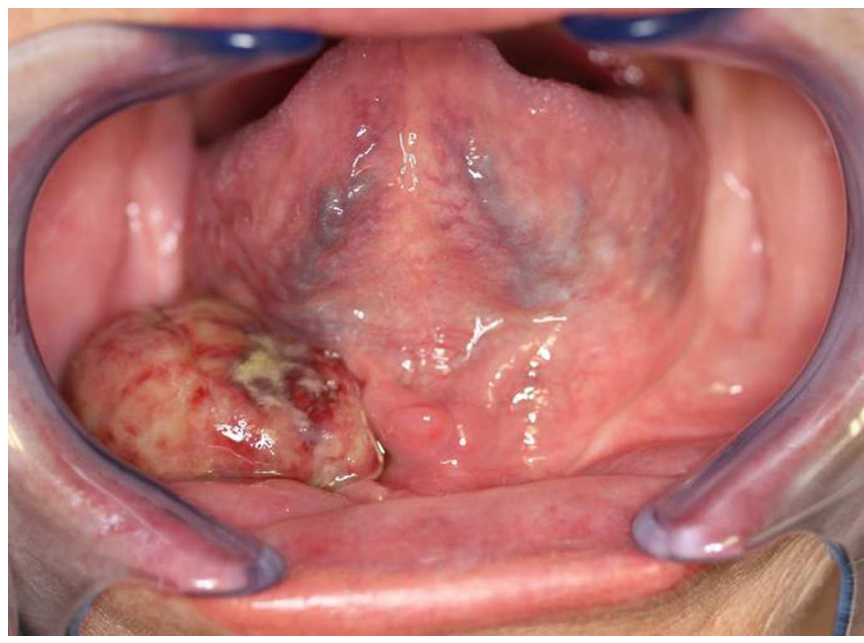

Fig. 5. Relapse of the lesion 40 days after resection.

recurrence risk is higher in implanted patients than in nonimplanted patients ( $42 \%$ and $17.5 \%$, respectively) [6]. The uniqueness of this clinical case lies in a possible correlation between classical peri-implantitis and the concomitant occurrence of a giant-cell granuloma. Similar cases were found with mandibular implants [7].

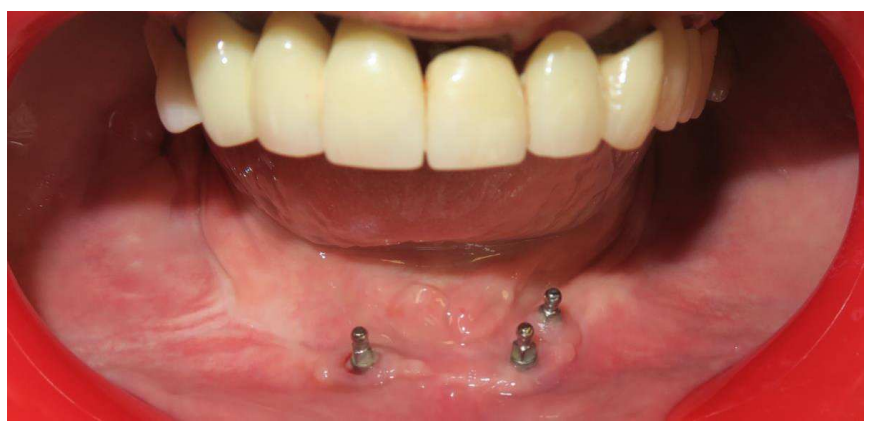

Fig. 6. Final mucosal healing after the installation of three minimandibular implants. There was no recurrence 1 year after the second procedure.

Peri-implantitis could be the cause of a specific lesion such as a giant-cell granuloma.

The inflammatory nature of the tissues bordering the implant and the implant mobility (sign of incomplete osseointegration) directed us to a removal of implants. This second resection was successful. A single alveolar curettage was sufficient. A more invasive peripheral osteoplasty would be useless [8]. The hypothesized correlation between the peri- 
implantitis and the mucosal lesion is validated by the absence of recurrence after suppression of the local infection.

A literature review was conducted in 2016 listing cases of giant-cell granulomas associated with implants [9]. Of the 12 cases published between 2003 and 2012, it appears that: nine concern women and eight have a gingival exophytic form. of these 12 cases, five cases resulted in a recurrence and five lesions recurred after the resection. According to this study, giant-cell granulomas are considered to be a reactive gingival pathology, the etiology of which would be chronic irritation around the implant pillars, especially when there is decreased space between two implants. Clinical data and therapeutic management of the clinical case reported above correspond to the published data.

An intralesional corticosteroid injection could also have been an alternative treatment [10]. However, given the implant mobility and the patient's willingness, surgical solution was preferred. The stability of the bony base made it possible to employ a classic prosthetic rehabilitation of a completely removable supra-implant prosthesis restoring masticatory, aesthetic, and phonetic function.

\section{Conclusion}

The giant-cell granuloma is a pathology well described in the literature, especially in terms of its potential for relapses around implants. The clinical case showed a possible causeeffect relationship between a peri-implantitis and lesion onset. This case suggests the possible role of inflammation on the appearance of a giant-cell granuloma. More in-depth studies to assert this correlation are needed.

\section{References}

1. Lindhe J, Meyle J, On behalf of Group D of the european workshop on periodontology, peri-implant diseases: consensus report of the sixth european workshop on periodontology. J Clin Periodontol 2008;35:282-285.

2. Regezi JA, Sciubba JJ, Jordan RCK. Oral Pathology: Clinical Pathologic Correlations. Elsevier Health Sciences; 2012. 1143 p.

3. Feine JS, Carlsson GE, Awad MA, Chehade A, Duncan WJ, Gizani S, et al. The McGill consensus statement on overdentures. Mandibular two-implant overdentures as first choice standard of care for edentulous patients. Int J Oral Maxillofac Implants 2002;17:601-602.

4. Bodner L, Peist M, Gatot A, Fliss DM. Growth potential of peripheral giant cell granuloma. Oral Surg Oral Med Oral Pathol Oral Radiol Endodontol 1997;83:548-551.

5. Chuong R, Kaban LB, Kozakewich H, Perez-Atayde A. Central giant cell lesions of the jaws: A clinicopathologic study. J Oral Maxillofac Surg 1986;44:708-713.

6. Lester SR, Cordell KG, Rosebush MS, Palaiologou AA, Maney P. Peripheral giant cell granulomas: a series of 279 cases. Oral Surg Oral Med Oral Pathol Oral Radiol 2014;118:475-482.

7. Hirshberg A, Kozlovsky A, Schwartz-Arad D, Mardinger O, Kaplan I. Peripheral Giant Cell Granuloma Associated with Dental Implants. J Periodontol. 2003;74:1381-1384.

8. Eisenbud L, Stern M, Rothberg M, Sachs SA. Central giant cell granuloma of the jaws: Experiences in the management of thirtyseven cases. J Oral Maxillofac Surg 1988;46:376-384.

9. Moreau N, Renoux M, Ejeil A-L. Implantologie et pathologies de la muqueuse buccale: une revue de la littérature. Médecine Buccale Chir Buccale 2016;22:13-29.

10. Kermer C, Millesi W, Watzke IM. Local injection of corticosteroids for central giant cell granuloma. A case report. Int J Oral Maxillofac Surg 1994;23:366-368. 